\title{
Tratamento cirúrgico da fibrilação atrial. Procedimento do "labirinto": experiência inicial
}

\author{
Adib D. JATENE*, Eduardo SOSA*, Flávio TARASOUTCHI, Marcelo B. JATENE*, Pablo M. A. \\ POMERANTZEFF
}

JATENE, A. D.; SOSA, E.; TARASOUTCHI, F.; JATENE, M. B.; POMERANTZEFF, P. M. - Tratamento cirúrgico da fibrilação atrial. Procedimento do "labirinto": experiência inicial. Rev. Bras. Cir. Cardiovasc., 7(2):107-111, 1992.

RESUMO: O procedimento "labirinto" para o tratamento cirúrgico da fibrilaçāo atrial (FA) proposto por Cox, para pacientes com FA, foi realizado entre julho de 1991 e maio de 1992, em nove pacientes, sendo sete do sexo feminino, com idade variando entre 37 e 63 anos $(M=51,4$ anos). Oito pacientes tinham disfunçōes valvares reumáticas associadas (estenose mitral pura: seis pacientes; dupla disfunçāo mitral (DLM) um paciente e DLM e insuficiência trisúspide: um paciente). A FA foi classificada como crônica (mais de um ano de duraçāo) de natureza reumática. Um paciente nāo tinha disfunçāo valvar associada e a FA foi considerada como paroxística recorrente refratária e nāo reumática. O procedimento cirúrgico foi realizado com o auxílio da circulaçāo extracorpórea e consistiu na realizaçāo de múltiplas e previamente bem estabelecidas incisōes nos átrios, de modo a tornar impossível a um impulso elétrico, originando-se em qualquer ponto dos átrios, retornar ao ponto de origem sem se deparar com uma linha de sutura. Isso impediria a ocorrência de reentrada em qualquer parte dos átrios. As múltiplas incisōes sāo de tal foram realizadas que, adicionalmente, permitem que o impulso originado na regiảo do nó sinusal atinja o nó AV e chegue aos ventrículos, após ter percorrido um "labirinto" criado pelas incisōes. O procedimento foi complementado com comissurotomia mitral em seis pacientes, troca da valva mitral (TVM) em um paciente e TVM e plastia de DeVega (PdV) em valva tricúspide em um paciente.

O primeiro paciente foi submetido a revisão cirúrgica para hemostasia. Nos demais, não houve problemas intra-operatórios. O segundo e o terceiro paciente apresentaram taquicardia atrial paroxística entre o terceiro e quinto dias de pós-operatório (PO). Um deles apresentou, também, no $15^{\circ} \mathrm{dia}$. Em todos os casos, a arrítmia foi controlada com amiodarona EV; o último paciente (com TVM e PdV) teve óbito no $45^{\circ}$ dia de PO, por complicaçōes infecciosas. Os pacientes restantes obtiveram alta hospitalar sem drogas antiarrítmicas. Em um período de um a dez meses $(M=5,4)$, os pacientes estāo assintomáticos e o Holter mostra presença de ritmo atrial irregular permanente (com FC média de 70 a $80 \mathrm{bpm}$ ) com condução AV preservada; o ecodoppler mostra presença de contraçāo atrial eficiente. Nảo houve recorrências de FA e nenhum dos pacientes.

Em conclusāo, podemos admitir que, a curto prazo, a técnica do "labirinto" na FA em reumáticos restaurou a contraçāo atrial organizada e controlou a FC. Assim, pode contribuir para reduçāo de fenômenos trombo-embólicos. Maior número de pacientes deve ser observado durante tempo prolongado para avaliaçāo da eficácia do procedimento.

DESCRITORES: fibrilaçāo atrial, cirurgia; "labirinto", técnica cirúrgica.

\footnotetext{
Trabalho realizado no Instituto do Coraçāo do Hospital das Clínicas da Faculdade de Medicina da Universidade de São Paulo. Sáo Paulo, SP, Brasil. Apresentado ao $19^{\circ}$ Congresso Nacional de Cirurgia Cardíaca. São Paulo, SP, 7 a 9 de maio, 1992.

* Do Instituto do Coraçáo do Hospital das Clínicas da Faculdade de Medicina da Universidade de São Paulo.

Endereço para separatas: Adib D. Jatene. Av. Dr. Enéas Carvalho de Aguiar, 44. Conselho Diretor. 05403 São Paulo, SP, Brasil.
} 
JATENE, A. D.; SOSA, E.; TARASOUTCHI, F.; JATENE, M. B.; POMERANTZEFF, P. M. - Tratamento cirúrgico da fibrilaçāo atrial.

Procedimento do "labirinto": experiência inicial. Rev. Bras. Cir. Cardiovasc., 7(2):1992.

\section{INTRODUÇĀO}

A fibrilaçāo atrial (FA) é arritmia freqüente acima dos 60 anos de idade $1,2,7,10,11,14,17$ e muito comum em portadores de estenose mitral, podendo ocorrer, no entanto, em pessoas mais jovens e sem cardiopatia.

Dados de literatura mostram que sua prevalência na populaçāo geral varia de $0,15 \%$ a $1 \%{ }^{5-0}$, sendo que acima dos 60 anos varia de $8 \%$ a $17 \%$ e na doença mitral chega a $79 \%$.

Embora seja possível conviver com a FA sob medicação, existem pelo menos três inconvenientes: 1) pulso irregular com aumento da freqüência ventricular; 2) redução do enchimento diastólico com decorrente distúrbio hemodinâmico e possibilidades de regurgitaçāo valvar; 3 ) possibilidades de tromboembolismo.

A reversāo para ritmo sinusal pode ser obtida por fármacos, ou por desfibrilaçāo elétrica. Reverter ao ritmo sinusal é um problema e manter esse ritmo é outro. Sabe-se que grande parte dos doentes volta a apresentar a arritmia e, em muitos, ela é permanente. Acresce a possibilidade de episódios paroxísticos, que, em pacientes com alguma disfunçāo cardíaca, pode levá-los com certa freqüência a serviços de emergência para se proceder à desfibrilaçăo elétrica.

A possibilidade de conviver com fibrilação atrial, com freqüência dentro de limites aceitáveis nāo elimina o risco de tromboembolismo. De acordo com FISHES ${ }^{\circ}$, cerca de $33 \%$ dos episódios de tromboembolismo estāo associados à FA, dos quais $60 \%$ resultam em morte ou seqüela neurológica permanente.

Diante de dificuldade em se reverter de forma permanente ao ritmo sinusal, algumas tentativas por cateterismo ou cirurgia foram tentadas. Mais recentemente COX et alii ${ }^{11-14}$ desenvolveram uma técnica que pretende interromper todos os potenciais circuitos de reentrada, restaurar o controle dos batimentos feitos pelos átrios e permitir que o estímulo atrial ative pela via normal o miocárdio ventricular.

Nossa experiência com esta técnica consta de 9 pacientes operados, objeto deste trabalho.

\section{CASUÍSTICA E MÉTODOS}

Entre julho de 1991 e maio de 1992, foram operados nove pacientes portadores de FA crônica, com idade entre 37 e 63 anos (média 51,4 anos). Quanto ao sexo, sete eram mulheres e dois homens.

Havia lesāo mitral associada em oito pacientes, sendo seis com estenose mitral pura e dois com dupla lesāo mitral.

Os oito pacientes com lesōes sobre a valva mitral apresentavam-se em classe funcional II/II, com indicação de tratamento cirúrgico da valvopatia.

Um paciente nāo tinha disfunçăo valvar associada e a FA foi considerada como paroxística recorrente refratária e năo reumática (Tabela 1 ).

A operaçāo é realizada através de toracotomia médio-esternal com emprego de circulação extracorpórea (CEC), hipotermia sistêmica a $25^{\circ} \mathrm{C}$ e parada cardíaca por soluçāo cardioplégica cristalóide inicialmente contendo $25 \mathrm{mEq} / \mathrm{d}$ de potássio e, após 25 a 30 minutos, novas infusōes de soluçāo cardioplégica de manutenção, contendo $7 \mathrm{mEq} / \mathrm{l}$ para adequada proteçāo do miocárdio.

Antes da colocaçāo das cânulas para drenagem venosa, procede-se a cuidadosa dissecção do coraçāo, segundo a descriçāo de $\mathrm{COX}^{3}$. A veia cava

TABELA 1

CASUISTICA

\begin{tabular}{|c|c|c|c|c|c|}
\hline CASO & IDADE & SEXO & $\begin{array}{l}\text { VALVOPATIA } \\
\text { ASSOCIADA }\end{array}$ & TIPO-FA & $\begin{array}{l}\text { CIRURGIAS } \\
\text { ASSOCIADAS }\end{array}$ \\
\hline 1 & 47 & $M$ & DLM & CRÔNICA & TVM+TROMB. \\
\hline 2 & 53 & $F$ & EM & CRÔNICA & COMISS. \\
\hline 3 & 37 & $\mathbf{F}$ & EM & CROQ̂NICA & COMISS. \\
\hline 4 & 62 & F & EM & CRỐNICA & COMISS. \\
\hline 5 & 57 & F & DLM+IT & CRÔNICA & TVM+DeVegaT \\
\hline 6 & 63 & $M$ & - & PAROXIST. & - \\
\hline 7 & 52 & $\mathrm{~F}$ & EM & CRÔNICA & COMISS.+TROMB. \\
\hline 8 & 42 & $\mathrm{~F}$ & EM & CRÔNICA & cOMISS. \\
\hline 9 & 50 & $\mathbf{F}$ & EM & CRÔNICA & COMISS.+TROMB. \\
\hline
\end{tabular}

$\mathrm{DLM}=$ dupla lesão mitral; $\mathrm{EM}=$ estenose mitral; IT = insuficiência tricúspide; $\mathrm{TVM}=$ troca valva mitral; COMISS = comissurotomia mitral; DeVega

$\mathrm{T}=$ plástica de DeVega valva tricúspide; TROMB = trombectomia de átrio esquerdo. 
JATENE, A. D.; SOSA, E.; TARASOUTCHI, F.; JATENE, M. B.; POMERANTZEFF, P. M. - Tratamento cirúrgico da fibrilaçāo atrial. Procedimento do "labirinto": experiência inicial. Rev. Bras. Cir. Cardiovasc., 7(2):1992.

superior é liberada até a veia ázigos. A reflexão do pericárdio visceral entre a artéria pulmonar direita e a veia pulmonar direita (à direita da veia cava superior) e parede posterior do átrio esquerdo entre a aorta e a veia cava superior deve ser amplamente seccionada, liberando-se totalmente a parte superior e posterior do átrio esquerdo. Também o ligamento triangular deve ser amplamente seccionado, ficando - coração preso apenas pelas artérias e veias, inteiramente liberado das reflexōes pericárdicas. A dissecção inclui também a liberação da aorta e do tronco pulmonar. A canulação da aorta é feita pela técnica habitual, na aorta ascendente à altura da reflexão pericárdica e as cânulas venosas introduzidas nas veias cavas, sendo que a superior à altura da veia ázigos. Iniciada a CEC, é introduzida cânula para cardioplegia na aorta ascendente.

As incisōes atriais, conforme a seqüência descrita por $\mathrm{COX}^{3}$ visam isolar faixas dos átrios e feitas de tal forma a obrigar o estímulo atrial percorrer um labirinto de tecido atrial com extensão insuficiente para permitir que os circuitos de reentrada se completem. Nos nossos casos, a aurícula esquerda foi ressecada, bem como a aurícula direita.

O tratamento da valva mitral foi feito conforme o tipo de lesão, aproveitando-se a incisão inicial no topo do átrio esquerdo, que se continuou pelo septo interatrial. Utilizou-se, também, conforme a técnica original, crioblação próxima aos anéis mitral e tricúspide e ao nível do seio coronário.

Em seis pacientes foi feita comissurotomia mitral e, em dois, a valva mitral foi substituída por prótese biológica de pericárdio bovino. Em um desses pacientes associou-se plastia de deVega em valva tricúspide (Tabela 1).

A recomposição dos átrios foi feita com fio de Prolene 000 em sutura contínua por chuleio simples.

\section{RESULTADOS}

$O$ tempo de CEC variou de 2 horas e 10 minutos a 3 horas e 30 minutos (média $=2 \mathrm{~h} .51 \mathrm{~min}$ ). A soluçäo cardioplégica, repetida a cada 25 a 30 minutos, foi sempre aspirada para fora do circuito extracorpóreo e seu volume de infusāo variou de 750 a $1440 \mathrm{ml}$ (média $=1105 \mathrm{ml}$ ). Observou-se diminuição progressiva do tempo de CEC com o acúmulo da experiência, bem como do tempo de pinçamento de aorta e do volume de soluçāo cardioplégica utilizada.

Em três pacientes existia trombose atrial esquerda e os trombos foram retirados em sua base de implantaçăo.
Um dos pacientes foi reoperado por sangramento e os demais evoluíram sem maiores complicaçōes no período de pós-operatório imediato.

Dois pacientes tiveram episódios de taquicardia atrial paroxística no $3^{\ell}$ e $5^{\ell}$ dias de pós-operatório, com repetiçāo do episódio no $15^{\circ}$ dia em um deles. A reversăo foi facilmentē obtida com infusāo de amiodarona intravenosa.

Nāo houve mortalidade operatória ou hospitalar, dentro dos primeiros 30 dias. Um paciente faleceu no segundo mês, em conseqüência de complicaçōes infecciosas.

Nenhum paciente retornou ao ritmo de FA no período de observação médio de 5,4 meses ( 1 a 10 meses).

A avaliação ecocardiográfica tardia demonstrou contração atrial eficiente e o Holter mostrou presença de rítmo atrial alto, regular, com extra-sístoles atriais ocasionais e freqüência cardíaca média oscilando de 70 a 80 batimentos por minuto, com conduçāo atrioventricular preservada (Tabela 2).

TABELA 2

RESULTADOS

\begin{tabular}{cccc}
\hline CASO & $\begin{array}{c}\text { ATIVIDADE ATRIAL } \\
\text { ELÉTRICA }\end{array}$ & MECÂNICA & $\begin{array}{c}\text { COMPLICAÇÕES } \\
\text { PÓS-OPERATÓRIAS }\end{array}$ \\
\hline 1 & RAR & PRESENTE & SANGRAMENTO-REOP. \\
2 & RAR & PRESENTE & TAQ.ATRIAL $3^{\circ}$ PO. \\
3 & RAR & PRESENTE & TAQ.ATRIAL 50 PO. \\
4 & RAR & PRESENTE & \\
5 & RAR & PRESENTE & ÓBITO NO $45^{\circ}$ PO \\
6 & RAR & PRESENTE & - \\
7 & RAR & PRESENTE & - \\
8 & RAR & PRESENTE & - \\
9 & RAR & PRESENTE & - \\
\hline
\end{tabular}

RAR = ritmo atrial regular;

\section{COMENTÁRIOS}

O insucesso do tratamento medicamentoso da FA tem estimulado tentativas mais agressivas. Mapeamento eletrofisiológico utilizando, simultaneamente, 208 eletrodos bipolares ${ }^{4}$, permitiu detectar mecanismos de reentrada particularmente próximos às veias cavas e pulmonares e próximos às aurículas e parede livre do átrio direito, levando por mecanismos diferentes à FA.

Isto explica porque o simples isolamento do átrio esquerdo proposto por WILLIAMS, et alii ${ }^{18}$, em 1980 , não conseguiu resolver o problema. A possibilidade do átrio esquerdo isolado continuar fibrilando mantém o risco de tromboembolismo. 
JATENE, A. D.; SOSA, E.; TARASOUTCHI, F.; JATENE, M. B.; POMERANTZEFF, P. M. - Tratamento cirúrgico da fibrilação atrial. Procedimento do "labirinto": experiência inicial. Rev. Bras. Cir. Cardiovasc., 7(2):1992.

A produção de bloqueio $A V$ total por ablação do feixe de His por criotermia, realizada em 1973, ou por cateterismo, complementado pelo implante de marcapasso ${ }^{15,18}$, não só trouxe um novo problema, como manteve a fibrilação dos átrios. Apenas os ventrículos voltam a ter ritmo regular, comandado por marcapasso.

Em 1985, GUIRAUDON et alii ${ }^{\circ}$ introduziram a técnica do corredor, em que uma tira do septo atrial ligava o nó sinoatrial ao nó atrioventricular. Essa técnica, embora permita o comando sinusal dos ventrículos, elimina o sincronismo atrioventricular e mantém a FA. Por isso, a evolução para uma técnica em que o estímulo atrial circule por um labirinto composto de faixas de músculo, cuja extensão impeça que os circuitos de reentrada se completem e que mantenha não só a contração atrial, mas também o sincronismo atrioventricular, era de se esperar. O procedimento proposto por COX et alii ${ }^{3-6}$ pretende cumprir esses objetivos.

A nossa experiência confirma os achados de CoX et alii ${ }^{3-6}$.

Os pacientes obtiveram alta sem medicação antiarrítmica e o óbito observado foi conseqüente a complicaçōes infecciosas.

Deve-se ressaltar que, na série de COX et alii ${ }^{3-6}$ apenas um paciente apresentava disfunção mitral, portanto, seus pacientes podem ser considerados não reumáticos. Ao contrário, oito dos nossos nove pacientes tinham lesōes reumáticas da valva mitral, que exigiram tratamento simultâneo. Este fato é importante, pois a presença da disfunçāo valvar reumática cria um substrato diferente daquele no qual Cox testou a técnica do "labirinto". Como é sabido, o tamanho dos átrios, particularmente o esquerdo, é maior quando há presença de lesão valvar associada.

Um aspecto interessante e que certamente merecerá maiores consideraçōes no futuro, é a secção sistemática da artéria do nó sinoatrial. Isto poderia conduzir a disfunçāo sinusal iatrogênica e justificar irregularidades no rítmo atrial. Certamente, no futuro esta variável será considerada.

Outro aspecto a se considerar é o tamanho do átrio esquerdo, já que, em átrios muito aumentados, a utilizaçāo da técnica original pode deixar faixas de tecido atrial largas, potencialmente capazes de permitir que a reentrada se complete e recidivando assim a FA. Nenhum dos nossos pacientes apresentava átrio extremamente volumoso, portanto com necessidade de modificação da técnica como a ressecção ou plicatura da parede atrial. Nāo ocorreu, em nenhum dos pacientes, recorrência de episódios de FA e, em todos, foi possível demonstrar. por ecocardiografia, contração atrial eficiente.

A eficácia do procedimento a longo prazo deve aguardar maior número de casos e tempo maior de observação.

JATENE, A. D.; SOSA, E.; TARASOUTCHI, F.; JATENE, M. B.; POMERANTZEFF, P. M. A. - Surgical treatment of atrial fibrillation with "maze" procedure: initial experience. Rev. Bras. Cir. Cardiovasc., 7(2):107-111, 1992.

ABSTRACT: The "maze" procedure for surgical treatment of chronic atrial fibrillation (AF) described by Cox was performed in 9 patients from July 91 to May 92; 7 were female and the ages range from 37 to $63 y$ $(51,4 y)$. Eight patients had surgical rheumatic valve disfunction (mitral stenosis in 6 ; mitral double disfunction in 2 being 1 with associated tricuspid regurgitation) and 1 had recurrent paroxicistic AF with no valve disfunction. Surgical treatment was performed following the technique described by Cox and the surgery was completed with 6 mitral comissurotomies and 2 mitral valve replacements.

Three patients had left atrial thrombosis. There were no immediate deaths and 1 patient died in the 45 th day with infeccious complications. The first patient required reoperation for bleeding review. Second and 3rd patients presented transitoryatrial tachycardia in $3 \mathrm{rd}$ and 5 th day, controlled with intravenous amiodarone. No other complications were observed. In a mean follow up period of $5,4 \mathrm{~m}(1$ to $10 \mathrm{~m})$, all patients were in regular atrial rhythm without antiarrhythmic drugs. Effective atrial contraction was demonstrated by $\mathrm{ECHO}$ in all patients and no one returned to AF. In conclusion, this initial follow up showed good results in rheumatic AF and more clinical observation is necessary to a definitive evaluation.

DESCRIPTORS: atrial fibrilation, surgery: "maze" procedure. 
JATENE, A. D.; SOSA, E.; TARASOUTCHI, F.; JATENE, M. B.; POMERANTZEFF, P. M. - Tratamento cirúrgico da fibrilação atrial. Procedimento do "labirinto": experiência inicial. Rev. Bras. Cir. Cardiovasc., 7(2):1992.

\section{REFERÊNCIAS BIBLIOGRÁFICAS}

CAMERON, A.; SCHWARTZ, M. J.; KRONMAL, R. A.; KOSINSKI, A. S. - Prevalence and significance of atrial fibrillation in coronary artery disease (CASS Registry). Am. J. Cardiol., 61:714-717, 1988.

COBLER, J. L.; WILLIAMS, M. E.; GREENLAND, P. Thyrotoxicosis in institutionalized elderly patients with atrial fibrillation. Arch.Intern.Med., 144:1758-1760, 1984.

COX, J. L. - The surgical treatment of atrial fibrillation. IV: Surgical technique. J. Thorac. Cardiovasc. Surg., 101:84-92, 1991.

4 COX, J. L.; CANAVAN, T. E.; SCHUESSLER, R. B.; CAIN, M. E.; LINDSAY, B. D.; STONE, C.; SMITH, P. K.; CORR, P. B.; BOINEAU, J. P. - The surgical treatment of atrial fibrillation. II:Intraoperative electrophsiologic mapping and description of the electrophysiologic basis of atrial flutter and atrial fibrillation. J. Thorac. Cardiovasc. Surg., 101:406426, 1991.

COX, J. L.; SCHUESSLER, R. B.; BOINEAL, J. P. - The surgical treatment of atrial fibrillation. I:Summary of the current concepts of the mechanism of atrial flutter and atrial fibrillation. J. Thorac. Cardiovasc. Surg., 101:402-405, 1991.

6 COX, J. L.; SCHUESSLER, R. B.; D'AGOSTINO Jr., H. J.; STONE, C. M.; CHANG, B. C.; CAIN, M. E.; CORR, P. B.; BOINEAU, J. P. - The surgical treatment of atrial fibrillation. III: Development of definitive surgical procedure. J. Thorac. Cardiovasc. Surg., 101:569-583, 1991.

DIAMONTOPOULOS, E. J.; ANTHOPOULOS, L.; NANAS, S.; MALIARAS, G.; CHRISOS, D.; MOULOPOULOS S. D. - Detection of arrhythmias in a representative sample of the Athens population. Eur. Heart J. 8(Supl.D):17-19, 1987.

8 FISCHER, C. M.; - Embolism in atrial fibrillation. In: KULBERTUS, H. E.; OLSSON, S. B.; SCHLEPPER, M. (eds.) Atrial fibrillation. Sweden, A.B.Hassle, 1982. p.192-210.

9 GUIRAUDON, G. M.; CAMPBELL, C. S.; JONES, D. L.; McLELLAN, J. L.; McDONALD, J. L. - Combined sino-atrial node atrio-ventricular node isolation: a surgical alternative to His bundle ablation in patients with atrial fibrillation. Circulation, 72 (Parte 3): 220 (Resumo).

KIMATA, S.; KANEKO, N.; NAKAMURA, K.; AOSAKI, M.; TAKAHASHI, S.; KONDO, M.; HIROSAWA, K.; SEKIGUCHI, M.; KASANUKI, H. - Natural history of atrial fibrillation. Heart Vessels, 2 (Supl.):14-23, 1987.

MARTIN, A.; BENBOW, L. J.; BUTROUS, G. S.; LEACH, C.; CAMM, A. J. - Five-year follow-up of 101 elderly subjects by means of long-term ambulatory cardiac monitoring. Eur. Heart J., 5:592-596, 1984.

ONUNDARSON, P. T.; THORGEIRSSON, G.; JONMUNDSSON, E., SIGFUSSON, N.; HARDARSON, T. - Chronic atrial fibrillation. Epidemiologic features and 14 year follow-up: a case control study. Eur. Heart J., 8:521-527, 1987.

SAVAGE, D. D.; GARRISON, R. J.; CASTELLI, W. P.; McNAMARA, P. M.; ANDERSON, S. J.; KANNEL. W. B.; FEILEIB, M. - Prevalence of submitral (anular) calcium and its correlates in a general populationbased sample (the Framingham Study). Am. J. Cardiol., 51:1375-1378, 1983.

TAMMARO, A. E.; RONZONI, D.; BONACCORSO, O.; BUTTE, M.; CARELLA, G. R.; COLOMBO, A. M.; COTTINO, M.; FRUSTAGLIA, A. GEMMELLARO, P.; PICCEO, M. T.; TREZZA, L.; TRICELLA, O. - La aritmie nell anziano. Minerva Med., 74:1313-1318, 1983.

SCHEINMAN, M. M.; MORADY, F.; HESS, D. S.; GONZALEZ, R. - Catheter-induced ablation of the atrioventricular junction to control refractory supraventricular arrhytmias. JAMA, 248:851-855, 1982.

SCHEINMAN, M. M. \& EVANS-BELL, T. - Catheter ablation of the atrioventricular junction: a report of the percutaneous mapping and ablation registry. Circulation, 70:1024-1029, 1984.

17 TRESEDER, A. S.; SASTRY, B. S.; THOMAS, T. P.; YATES, M. A.; PATHY, M. S. - Atrial fibrillation and stroke in elderly hospitalized patients. Age Aging, 15:89-92, 1986

18 WILLIAMS, J. M.; UNGERLEIDER, R. M.; LOFLAND, G. K.; COX, J. L. - Left atrial isolation: a new technique for the treatment of supraventricular arrhytmias. J. Thorac. Cardiovasc. Surg., 80:373-380, 1980. 